\title{
Hypereosinophilic syndrome with large intracardiac thrombus
}

\author{
Kian-Guan Lee ${ }^{1}$, MBBS, MRCP, Matthew Bingfeng $\underline{\text { Chuah }}^{2}$, MBBS, Hak-Chiaw Tang $^{3}$, MRCP, FAMS, \\ Terrance Siang Jin $\underline{\text { Chua }}^{4}$, FRCP, FAMS
}

\begin{abstract}
As the nonspecific clinical presentation of hypereosinophilic syndrome (HES) may mimic many multisystemic diseases, it often presents as a diagnostic challenge. Herein, we report the case of a 60-year-old man who presented with progressive heart failure symptoms and eosinophilia. Despite extensive diagnostic evaluation, no underlying cause was found. Transthoracic echocardiography revealed a large left ventricular thrombus, which is suggestive of hypereosinophilic cardiac involvement. The patient was started on steroids and responded clinically and haematologically.
\end{abstract}

Keywords: cardiomyopathy, eosinophilia, hypereosinophilic syndrome, intracardiac thrombus

\section{INTRODUCTION}

Hypereosinophilic syndrome (HES) is a disorder characterised by persistent eosinophilia with a heterogeneous group of clinical manifestations. Cardiac manifestation is noted in more than $50 \%$ of patients with HES and is the major cause of morbidity in such patients. ${ }^{(1)}$ Herein, we describe the case of an HES patient who presented with decompensated heart failure, discuss the typical echocardiographic features of hypereosinophilic cardiac involvement and provide a summary of the current medical management of the condition.

\section{CASE REPORT}

A 60-year-old man presented with a two-week history of progressive dyspnoea, reduced effort tolerance and ankle swelling. His past medical history included dyslipidaemia, coronary artery disease treated via bypass grafting, and recurrent scarred ventricular tachycardia treated via implantation of an automated implantable cardioverter-defibrillator. The patient also had a history of rash, which was examined at the National Skin Centre, Singapore. On physical examination, he appeared lethargic and dyspnoeic. Ascites and peripheral oedema, as well as bilateral basal crepitation on lung auscultation, were found. Electrocardiography (ECG) showed sinus rhythm, incomplete left bundle branch block with premature ventricular complexes, and nonspecific T-wave inversions (Fig. 1). The patient was initially managed as a case of decompensated cardiac failure in view of his underlying ischaemic heart disease. However, he did not respond to standard anti-failure therapy. Coronary angiography of the patient showed patent bypass grafts. Based on previous medical notes, he had a history of hypereosinophilic cellulitis on skin biopsy in 2004, but he subsequently defaulted on follow-up. Our laboratory investigations of the patient demonstrated leucocytosis $\left(16.9 \times 10^{9} / \mathrm{L}\right)$ with marked eosinophilia (eosinophilia 53\%; absolute eosinophil count $9,000 / \mu \mathrm{L}$ ) and thrombocytopenia $(23,000 / \mu \mathrm{L})$. Further investigations did not suggest any evidence of an underlying cause of hypereosinophilia, such as connective tissue disease or parasitic infestation. There was no history suggestive of allergy, drug reactions or malignancy. Bone marrow study (i.e. trephine biopsy) revealed bone marrow that was normocellular with trilineage haematopoiesis and mild eosinophilia, but with no dysplastic or myeloproliferative

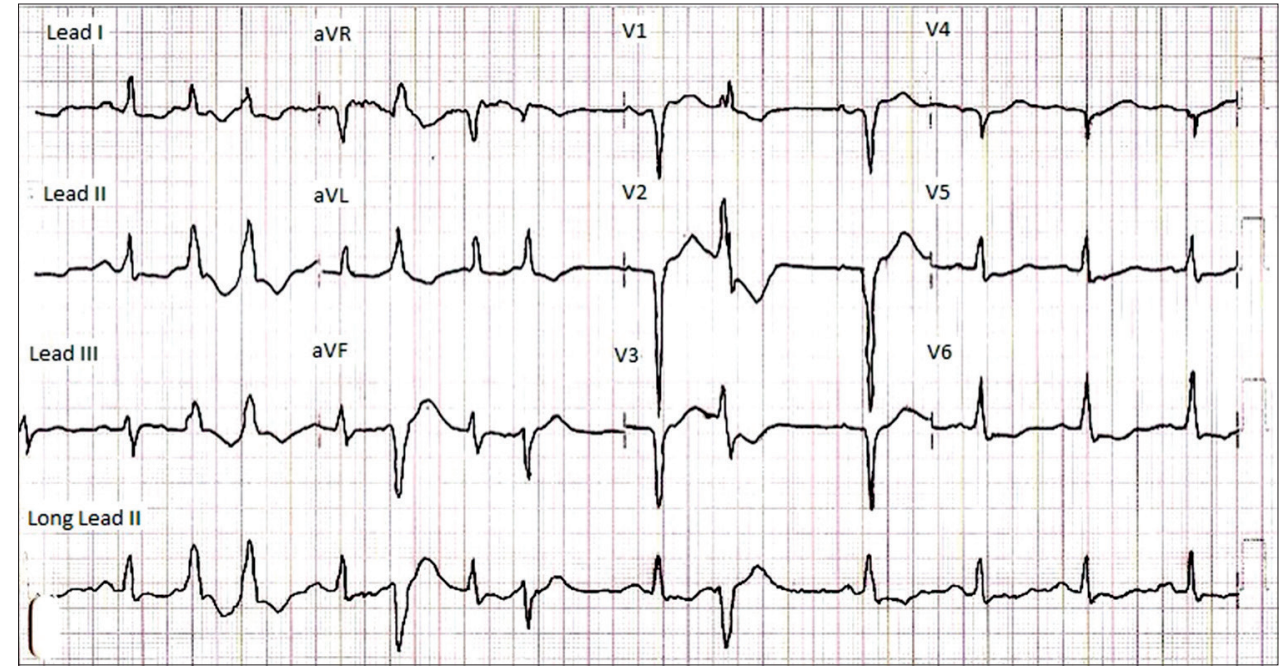

Fig. 1 ECG shows sinus rhythm, incomplete left bundle branch block with premature ventricular complexes, and nonspecific T-wave inversions.

${ }^{1}$ Department of Renal Medicine, ${ }^{2}$ Internal Medicine, Singapore General Hospital, ${ }^{3}$ National Heart Centre, Singapore

Correspondence: Dr Kian-Guan Lee, Senior Resident, Renal Medicine Department, Singapore General Hospital, Outram Road, Singapore 169608. kianguan.lee@mohh.com.sg 


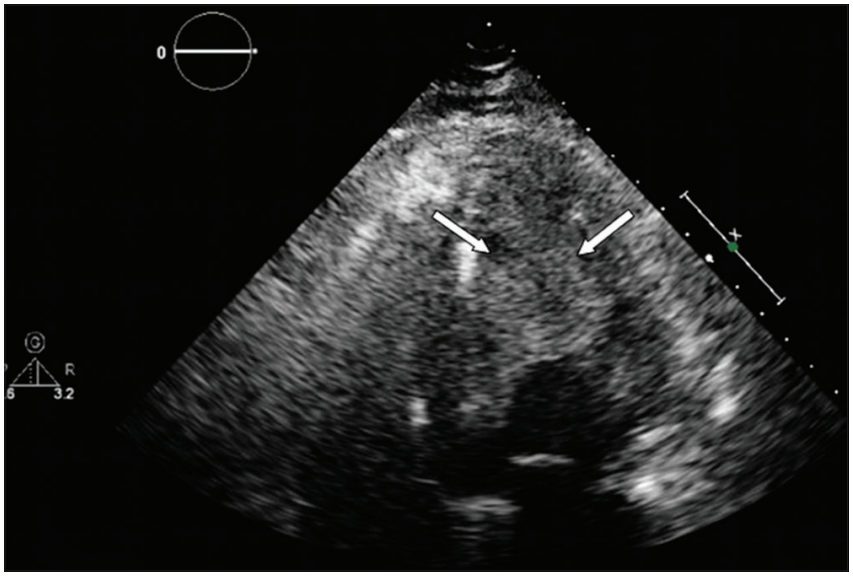

Fig. 2 Transthoracic echocardiogram shows a large echodense mass (arrows) in the mid to apical level of the left ventricular chamber, with the presence of apical hypokinesia, and no right ventricular clot.

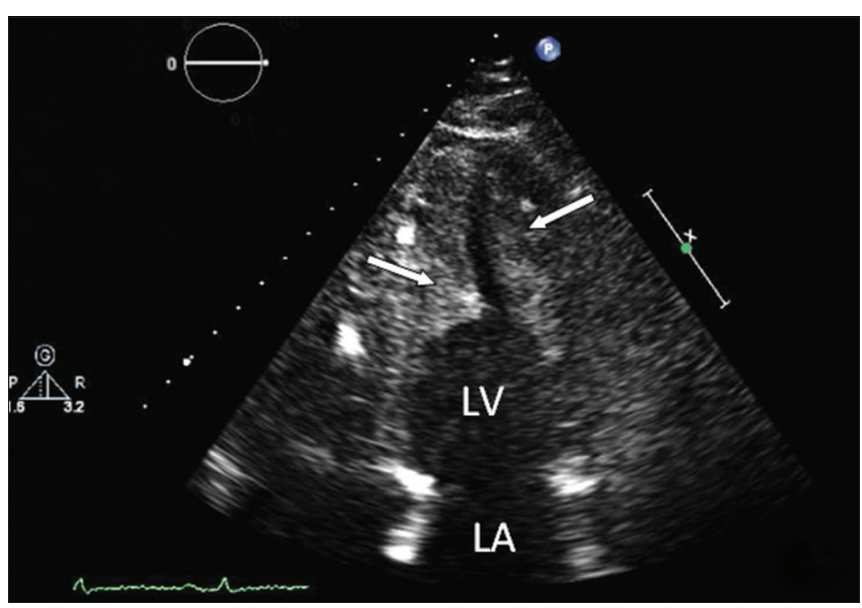

Fig. 4 Transthoracic echocardiogram shows a reduction in thrombus size three weeks after initiation of treatment (arrows), and no regional wall motion abnormality. LA: left atrium; LV: left ventricle.

features. Flow cytometry showed no evidence of any clonal lymphoproliferative disorder. Specifically, the FIP1L1-PDGFRA fusion gene transcript was not detected. A diagnosis of idiopathic HES was thus made.

Transthoracic echocardiography (TTE) showed normal ejection fraction with no regional wall motion abnormalities. A large mass adhering to the left ventricular endocardium was seen from the mid to apical level (Fig. 2). Mitral inflow revealed a restrictive Doppler filling pattern. The mass was found to be non-enhanced after left ventricular opacification with an echo-contrast agent (SonoVue, Bracco Diagnostics Inc, USA) (Fig. 3), which was suggestive of a thrombus. There was no significant valvular involvement and the echocardiographic appearance is typical of hypereosinophilic cardiac involvement.

The patient was commenced on steroids, and responded clinically and haematologically with normalisation of eosinophil count within a week. He was also anticoagulated with warfarin therapy for prevention against cardioembolism. His heart failure symptoms improved, and serial TTE on follow-ups after the first (Fig. 4) and second (Fig. 5) months showed progressive reduction in thrombus size.

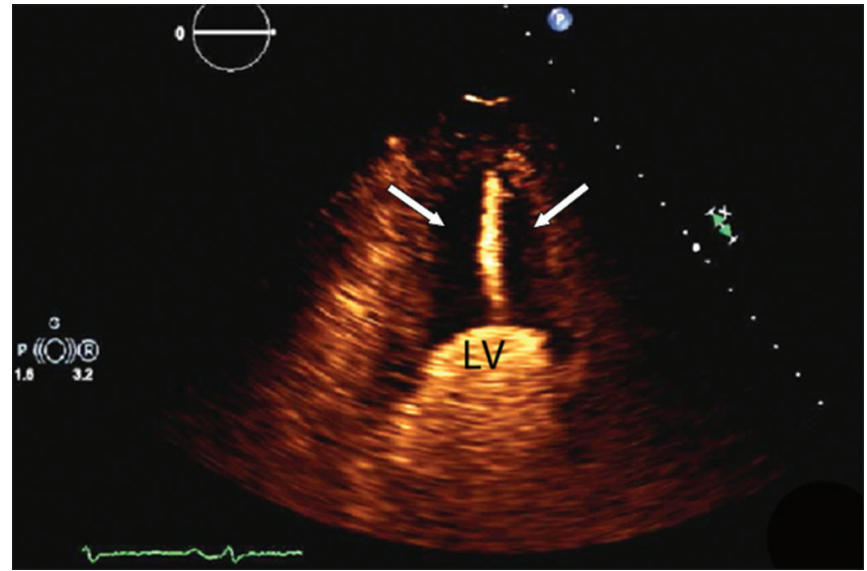

Fig. 3 SonoVue echo-contrasted transthoracic echocardiogram shows a large thrombus (arrows) attached to the thickened myocardium. LV: left ventricle.

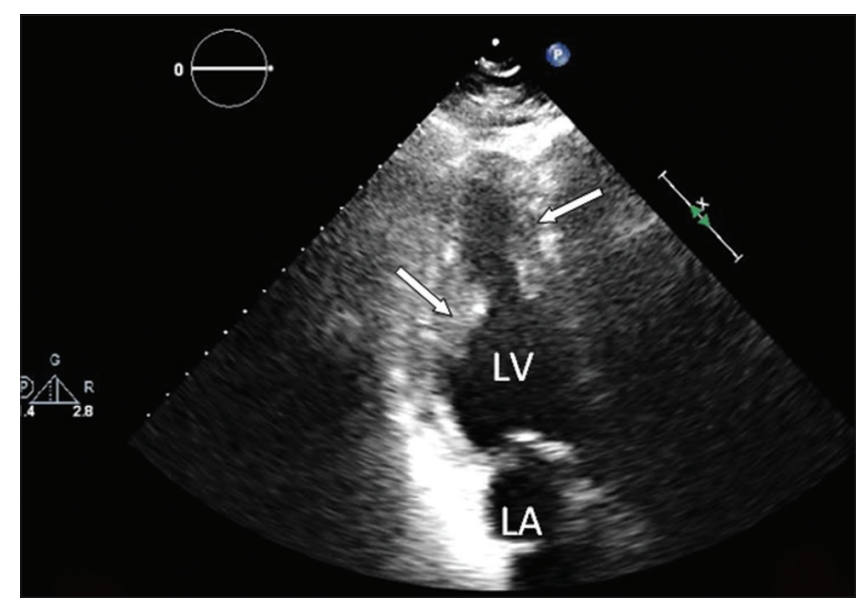

Fig. 5 Transthoracic echocardiogram shows a further reduction in thrombus size two months after treatment (arrows). LA: left atrium; LV: left ventricle.

\section{DISCUSSION}

We described the case of an HES patient who presented with a large intracardiac thrombus and symptoms of decompensated cardiac failure. Chusid et al defined idiopathic HES as blood eosinophilia (a) exceeding 1,500/ $\mathrm{L}$ for more than six months without other evident causes despite extensive diagnostic evaluation; and (b) leading to organ involvement as a result of eosinophilic infiltration. (2) Frequently involved systems and organs include the cardiovascular system, nervous system, lungs, gastrointestinal tract and skin. As there is no specific diagnostic test for HES, it remains a diagnosis of exclusion. The condition's nonspecific clinical presentation may also mimic other multisystemic diseases such as hypersensitivity vasculitis, Löffler's syndrome, eosinophilic leukaemia and myeloproliferative disorders with predominant eosinophilia. ${ }^{(3)}$

Cardiac involvement is seen in more than $50 \%$ of patients with HES, and a patient with HES usually presents with uni- or biventricular, pericardial or global myocardial involvement. ${ }^{(1)}$ It is associated with poor clinical outcome, with the majority of deaths related to cardiac complications and thromboembolic events. $^{(3)}$ The pathological process occurs in stages, beginning from eosinophilic infiltration, progressing to an intermediate 
thrombotic stage and eventually resulting in endomyocardial fibrosis, restrictive cardiomyopathy and progressive valvular incompetence. ${ }^{(4)}$ Blood hypercoagulability may contribute to the pathogenesis of thrombosis in HES, typically as a result of the release of von Willebrand factor and tissue factor, as well as factor XII activation by eosinophil granule proteins. ${ }^{(1)}$ Common findings in ECG include the presence of premature ventricular complexes, nonspecific T-wave inversions, and signs of left atrial and/or left ventricular enlargement. ${ }^{(1)}$ The hallmark echocardiographic finding, as described in this case, is the obliteration of the apex of the ventricles by laminar thrombus. The restrictive pattern and absence of ischaemic features on TTE suggest that this thrombus is unlikely to be of ischaemic origin.

Development of vascular and valvular damage may also lead to congestive cardiac failure. Contrast-enhanced magnetic resonance (MR) imaging, with its ability for tissue characterisation, is highly useful as a noninvasive diagnostic imaging technique for cardiac manifestation in HES, allowing for early diagnosis as well as the monitoring of treatment efficiency. ${ }^{(5)}$ However, in cases not accompanied by peripheral eosinophilia, endomyocardial biopsy remains the diagnostic gold standard for endocardial involvement in HES. ${ }^{(1)}$

Early recognition and diagnosis of HES are crucial to allow for appropriate treatment and optimise clinical outcome. Corticosteroids are considered in first-line therapy of HES, aiming to reduce peripheral and tissue levels of eosinophils, and prevent end-organ damage. In steroid-resistant cases, however, cytotoxic agents and interferon- $\alpha$ can be used. In recent years, evaluation of molecular analysis and lymphocyte clonality has been suggested to guide treatment regimen. Imatinib mesylate has been shown to be effective in the treatment of HES with FIP1L1-PDGFRA fusion gene mutation. ${ }^{(3)}$ For FIP1L1-PDGFRA fusion gene-negative patients, the newer mepolizumab is shown to be effective, resulting in corticosteroid-sparing treatment for HES. ${ }^{(6)}$

In conclusion, HES is an uncommon heterogeneous disease syndrome that requires a high clinical suspicion index so as to enable early diagnosis. Cardiac involvement of HES is associated with unfavourable clinical outcome and therefore timely medical treatment is crucial to prevent disease progression. Recent advances in diagnostic imaging and a better understanding of HES pathogenesis have resulted in noninvasive diagnostic opportunities and novel targeted therapies, respectively.

\section{REFERENCES}

1. Kleinfeldt T, Nienaber CA, Kische $S$, et al. Cardiac manifestation of the hypereosinophilic syndrome: new insights. Clin Res Cardiol 2010; 99:419-27.

2. Chusid MJ, Dale DC, West BC, Wolff SM. The hypereosinophilic syndrome: analysis of fourteen cases with review of the literature. Medicine (Baltimore)1975; 54:1-27.

3. Wilkins HJ, Crane MM, Copeland K, Williams WV. Hypereosinophilic syndrome: an update. Am J Hematol 2005; 80:148-57.

4. Salanitri GC. Endomyocardial fibrosis and intracardiac thrombus occurring in idiopathic hypereosinophilic syndrome. Am J Roentgenol 2005; 184:1432-3

5. Pillar N, Halkin A, Aviram G. Hypereosinophilic syndrome with cardiac involvement: early diagnosis by cardiac magnetic resonance imaging. Can J Cardiol 2012; 28:515.e11-3.

6. Rothenberg ME, Klion AD, Roufosse FE, et al. Treatment of patients with the hypereosinophilic syndrome with mepolizumab. N Engl J Med 2008; 358:1215-28. 